\title{
A phase I clinical study of VB4-845: Weekly intratumoral administration of an anti-EpCAM recombinant fusion protein in patients with squamous cell carcinoma of the head and neck
}

\author{
Glen C MacDonald' \\ Michèle Rasamoelisolo' \\ Joycelyn Entwistle' \\ Jeannick Cizeau' \\ Denis Bosc' \\ Wendy Cuthbert ${ }^{2}$ \\ Mark Kowalski \\ Maureen Spearman' \\ Nick Glover ${ }^{2}$ \\ 'Viventia Biotech Inc., Winnipeg, \\ Manitoba, Canada; ${ }^{2}$ Viventia Biotech \\ Inc., Mississauga, Ontario, Canada
}

Correspondence: Maureen Spearman Viventia Biotech Inc., 147 Hamelin St., Winnipeg, Manitoba, Canada R3T 3ZI

$\mathrm{Tel}+\mathrm{I} 204478$ I023

Fax + I 204452772 I

Email mspearman@viventia.com

\begin{abstract}
VB4-845 is a scFv-Pseudomonas exotoxin A fusion construct that targets epithelial cell adhesion molecule (EpCAM). A phase I trial was conducted to determine the maximum tolerated dose (MTD) of VB4-845 when administered as weekly intratumoral (IT) injections to patients with squamous cell carcinoma of the head and neck (SCCHN). Secondary objectives included the evaluation of the safety, tolerability, pharmacokinetic profile, and immunogenicity, and a preliminary assessment of tumor response. Twenty patients with advanced, recurrent SCCHN were treated weekly for four weeks in ascending dose cohorts of 100, 200, 330, 500, 700 , and $930 \mu \mathrm{g}$. The MTD was established as $930 \mu \mathrm{g}$ with a dose limiting toxicity of elevated liver enzymes in two of five patients. VB4-845 therapy was well tolerated with common treatment-related adverse events of injection site reactions, fever, gastrointestinal disorders, and elevated liver enzyme levels. All patients developed antibodies to VB4-845 by the end of the study, but only seven patients had neutralizing antibodies. Preliminary efficacy data found $87.5 \%$ of EpCAM-positive patients had a positive response to VB4-845 therapy. Noninjected dermal metastases were also resolved in one patient. VB4-845 IT therapy is safe and feasible and warrants further clinical evaluation for the treatment of SCCHN
\end{abstract}

Keywords: EpCAM, phase I, immunotoxin, head and neck cancer, VB4-845

\section{Introduction}

Combined treatment regimes, such as chemoradiotherapy, have recently improved the prognosis of patients with squamous cell carcinoma of the head and neck (SCCHN) (Posner 2007); however response to second line treatment remains very low (León et al 2005). As such, new treatment options for these patients are urgently needed and a variety of molecules are currently under study (Haddad et al 2006; Bossi et al 2007; Le Tourneau et al 2007).

The use of monoclonal antibodies and immunotoxins offers target-specific and increasingly effective treatment options where traditional therapies have failed (Robert et al 2001; von Mehren et al 2003; MacDonald and Glover 2005; Bonner et al 2006; Pastan et al 2007; Gebbia et al 2007; Blick et al 2007). One such target, epithelial cell adhesion molecule (EpCAM or CD326), whose normal function is to mediate epithelium-specific, homotypic cell-cell adhesions (Litvinov et al 1994, 1997), is highly expressed in a variety of carcinomas (Balzar et al 1999; Winter et al 2003; Went et al 2004), including squamous cell carcinoma of the tongue (Yanamoto et al 2007), esophagus (Stoecklein et al 2006) and oral cavity (Laimer et al 2008), and thus represents an ideal target for immunotherapy.

VB4-845 is a recombinant fusion protein that combines the specificity of an antiEpCAM single chain variable fragment $(\mathrm{scFv})$ with the toxicity of Pseudomonas 
exotoxin A (ETA $\left.{ }_{252-608}\right)$ (Di Paolo et al 2003). Once bound to EpCAM via the $\mathrm{scFv}$ portion, the fusion construct is internalized through an endocytic pathway. An activated form of ETA is released into the cytosol following furin cleavage where it inhibits protein synthesis ultimately leading to cell death (Perentesis et al 1992; Pastan et al 2006). VB4-845 studies have previously shown significant growth inhibition of EpCAM-positive, human tumor xenografts of colorectal cancer, small cell lung cancer, and SCCHN in mice (Di Paolo et al 2003). Other clinical studies using systemic administration of immunotoxins have resulted in vascular leak syndrome (VLS), a nonspecific inflammatory response, as well as a dose-limiting immunogenic response (Siegall et al 1997; Pastan et al 2006). Therefore, we have chosen intratumoral (IT) administration (Rand et al 2000; Azemar et al 2003) to reduce the potential of VLS and minimize the effect of an anti-VB4-845 response. Drug concentrations were chosen based upon our preclinical results (Brown et al 2005). This phase I dose-escalation trial was conducted in patients with advanced, recurrent SCCHN, where VB4-845 was administered weekly for four consecutive weeks. The primary objective of this study was to determine the maximum tolerated dose (MTD) of VB4-845, while the secondary objectives evaluated the safety, tolerability, pharmacokinetic profile, immunogenicity, and a preliminary determination of tumor response.

\section{Patients and methods}

\section{Patient selection}

Patients 18 years of age or older, with histologically confirmed recurrent SCCHN following radiotherapy and/or chemotherapy, were eligible for enrollment. At least one bidimensional, measurable target lesion that was amenable to direct injection was required. Key inclusion criteria were a Karnofsky performance status of at least 70 and an estimated life expectancy of more than three months. Further inclusion criteria included demonstration of adequate hepatic function (alanine aminotransferase [ALT] and aspartate aminotransferase $[\mathrm{AST}] \leq 2.5$ times the upper limit of normal [ULN], and bilirubin levels $\leq 1.5$ times ULN); adequate renal function (serum creatinine $\leq 1.5$ times ULN, or creatinine clearance $\geq 60 \mathrm{~mL} /$ minute) and hematologic values required for inclusion were granulocytes $\geq 1500 / \mu \mathrm{L}$, platelets $\geq 100000 / \mu \mathrm{L}$, and hemoglobin $>8 \mathrm{~g} / \mathrm{dL}$. Key exclusion criteria included suspected or impending airway obstruction; suspected or documented carotid artery infiltration by the injectable tumor; history of HIV, hepatitis $\mathrm{C}$, hepatitis B, or brain metastasis; uncontrolled hypertension; a prior splenectomy; treatment within four weeks prior to screening with systemic corticosteroids, any other monoclonal antibody, investigational product, or radiotherapy; concurrent diseases that might affect interpretation of the study results, including clinically significant renal or hepatic disease; surgery with general anesthetic scheduled within four weeks of screening.

The study was conducted in accordance with the Declaration of Helsinki, International Conference on Harmonization guideline E6 (Good Clinical Practice), Title 21, Parts 50 and 56 of the United States Code of Federal Regulations and with the laws and regulations for the conduct of human clinical trials in Brazil, including the Institutional Review Board. Independent Ethics Committee (IEC) approval was obtained prior to initiating the study. Written informed consent was obtained from each patient before any study-related activity was performed.

\section{Study design and dose escalation}

The study was an open-label, multicenter, single-arm dose-escalating trial assessing the safety and tolerability of VB4-845 injected IT once weekly for four consecutive weeks. Twenty patients were treated in six dose cohorts, and were followed for four weeks after the last dose. The ascending modified Fibonacci dose cohorts were 100, 200, 330, 500, 700, and $930 \mu \mathrm{g}$. VB4-845 was supplied as a $1 \mathrm{mg} / \mathrm{mL}$ formulation and was diluted in phosphate buffered saline. Depending on the size and location of the tumor, VB4-845 was either administered from a single puncture site or from multiple sites in one $\mathrm{cm}$ increments evenly distributed throughout the tumor. Briefly, a 25 to 27 gauge needle attached to $1 \mathrm{cc}$ Luer-lock syringe was inserted into the base of the tumor at approximately a 45-degree angle. If blood appeared with a slight draw of the syringe, the needle was removed and reinserted in a different area of the tumor. Drug was only administered if no blood was observed. The suspension volume of PBS used for immunotoxin administration was equal to that required to bring the total dose volume to no more than $30 \%$ of the estimated volume of the tumor mass being injected. Depending on the size and location of the tumor, the injection was completed by tracking the product through the tumor from a single puncture site or by injecting the tumor from multiple sites, in $1 \mathrm{~cm}$ increments, in parallel rows approximately 0.5 to $1.0 \mathrm{~cm}$ apart and disbursing throughout the tumor. While the speed of injection was not dictated, the speed of injection generally depended upon the density of the tumor tissue and the ease of disbursing the study drug solution throughout the tumor while still 
allowing the retention of the study drug within the tumor. It should be noted that the selection of appropriate target tumors excluded those involving critical structures. The far majority of tumors were treatable without image guidance in the outpatient setting; however, image guidance with, for example, ultrasound or computed tomography (CT) was not discouraged.

At least three patients were enrolled in each dose cohort and dose escalation was permitted once two patients had completed the first two weeks of dosing without dose-limiting toxicity (DLT). The DLT was defined as the occurrence of adverse events related to treatment including: grade 4 toxicity for local reaction at the injection site, grade 4 flulike symptoms, grade 4 hematological toxicity, or any other nonhematological grade 3 toxicities with the exception of alopecia. If two patients experienced DLT at any dose, that dose was defined as the MTD.

Safety was evaluated by monitoring adverse events, hematology, blood chemistry/serology, and urinalysis, and regular physical examinations. The toxicity grade of an adverse event was classified according to the National Cancer Institute (NCI) common toxicity criteria (CTC), version 2.0. In order to fully characterize the local effects of IT VB4-845 administration, a local injection toxicity scale was included, based on the injection site reaction event in NCI CTC version 2.0 (see Table 2). Adverse events were judged to be treatmentrelated by the investigators if they were possibly, probably, or definitely related to VB4-845 administration.

\section{Patient evaluation}

Pretreatment screening evaluations were performed within two weeks of the baseline visit and included: history and physical examination; 12-lead electrocardiogram; identification, measurement, and digital photography of target and nontarget tumors; chest radiograph; urinalysis, blood collection for serum chemistry, and hematology and biopsy. Immunohistochemistry on tissue biopsies was performed to determine EpCAM expression in tumors. Baseline evaluations on day one of drug administration included assessment of Karnofsky performance status, and a physical examination. Prior to dosing on days $1,8,15$, and 22, and in the followup period (weeks 4, 6, and 8) vital signs were recorded, and urinalysis, blood biochemistry and hematological analyses were performed. Vital signs and adverse events were assessed for 24 hours post dose. Measurements of the target and nontarget tumors were performed prior to treatment and at weeks 4 and 8 using CT and caliper measurements and pain was also assessed. At the end of week 8 (or the patient's final visit) additional analyses included assessment of Karnofsky performance status, digital photographs of observable lesions, 12-lead electrocardiogram, and pregnancy test for nonsterile females of reproductive age.

\section{Pharmacokinetics}

Blood samples for pharmacokinetic analysis were taken on day one prior to drug injection and after dosing at 10 and 30 minutes, and at 1, 2, 4, 6, 12, and 24 hours, and prior to dosing on days $8,15,22$, and at the end of week four. The amount of VB4-845 in the plasma was evaluated by a potency assay using VB4-845-mediated cytotoxicity on the EpCAM-positive CAL-27 cell line (ATCC, Manassas, VA, USA). Patient samples or VB4-845 standards were incubated with pre-cultured CAL-27 cells at $37^{\circ} \mathrm{C}$ under $5 \% \mathrm{CO}_{2}$ for 72 hours, and washed with PBS-T (PBS with $0.5 \%$ polysorbate; Sigma Aldrich, St. Louis, MO, USA). Cell viability was determined using MTS reagent (tetrazolium compound; Promega, Madison, WI, USA) and the absorbance was read (Vmax, Molecular Devices, Sunnyvale, CA, USA). The amount of VB4-845 present in patient plasma was interpolated from the calibration curve of VB4-845 standards diluted in normal plasma.

\section{Immunogenicity}

Blood samples for assessing humoral immune reactivity to VB4-845 were collected as for the pharmacokinetic analysis on days $1,8,15,22,28$ prior to administration of VB4845 , and day 56. Antibody titers to the antibody fragment (anti-scFv), and the toxin portion (anti-ETA ${ }_{(252-608)}$ ) were measured using ELISA. Briefly, either purified ETA E $_{1-608}$ (Sigma Aldrich) or the human scFv moiety (4D5 scFv) were diluted to $14 \mathrm{nM}$ in carbonate buffer ( $\mathrm{pH}$ 9.6) and coated onto ELISA plates overnight at $4{ }^{\circ} \mathrm{C}$. The plates were washed and blocked, and serially-diluted patient plasma samples (PBS, $1 \%$ BSA) were added to the coated plates for 2 hours at room temperature. Antibodies to $\mathrm{ETA}_{1-608}$ or $4 \mathrm{D} 5 \mathrm{scFv}$ were detected by adding HRP-labeled goat antihuman-IgG, IgM, $\operatorname{IgA}(\mathrm{H}+\mathrm{L})$ antibodies (KPL, Gaithersburg, MD, USA) for 1 hour at RT, followed by washing and the addition of TMB (3,3',5,5' tetramethylenzidine; KPL) and the absorbance read (Vmax). The minimum required dilution of the plasma samples to avoid interference was $1 / 1000$ and the cut-off value for positivity was defined as $4 \mathrm{x}$ standard deviation of the O.D. of the blank buffer samples, ie, 0.1 O.D. To assess whether the patients had cross-reactive antibodies prior to treatment, the mean titer on Day 1 was expressed only as the geometric mean of the reciprocal dilution at 0.1 O.D. For all 
other days, the patient immune response measured following VB4-845 administration was expressed as the geometric mean of the reciprocal dilution at 0.1 O.D. after subtracting the pre-treatment sample (Day 1) O.D. value.

\section{Results}

\section{Patient characteristics}

A total of 20 patients were enrolled, all of whom had recurrent, advanced SCCHN (Table 1). Ninety percent of patients had received at least one cycle of chemotherapy and all had received radiotherapy. Patient enrollment was independent of tumor EpCAM status: 18 patients had EpCAM-positive tumors and two patients had EpCAM-negative tumors.

\section{Dose escalation, safety, and maximum tolerated dose}

The study progressed through doses of 100, 200, 330, 500, and $700 \mu \mathrm{g}$, and was terminated at $930 \mu \mathrm{g}$ upon reaching the MTD. Dosing was once weekly for four consecutive weeks, with a four week follow-up. Eight patients discontinued the study due to death unrelated to VB4-845 administration, withdrawal of consent, or adverse events.

All 20 patients reported at least one adverse event during the course of the study, with common adverse events including injection site reactions, tumor pain, gastrointestinal disorders,

Table I Patient characteristics

\begin{tabular}{ll}
\hline Total number of patients & $\mathbf{2 0}$ \\
\hline Median age in years (range) & $52(43-77)$ \\
Gender & 18 \\
Male & 2 \\
Female & \\
Ethnicity & 14 \\
Caucasian & 5 \\
Black & 1 \\
Other & \\
Karnofsky performance status & 5 \\
70 & 7 \\
80 & 6 \\
90 & 2 \\
I00 & \\
Prior therapy & 18 \\
Chemotherapy & 5 \\
I Cycle & 10 \\
2 Cycles & 3 \\
3 Cycles & 20 \\
Radiotherapy & \\
EpCAM expression & 18 \\
Positive & 2 \\
Negative &
\end{tabular}

Abbreviation: EpCAM, epithelial cell adhesion molecule. elevated liver enzymes, tumor bleeding, dehydration and fever. Treatment-related adverse events were reported by 16 patients $(80 \%)$ and are summarized in Table 2. Except for one instance of a grade 4 event (elevated gamma-glutamyltranspeptidase: GGT) in the $700 \mu \mathrm{g}$ cohort, all treatment-related adverse events were grade 3 or lower. The most frequent treatment-related adverse events were injection site reactions, which included tumor pain, edema, and/or erythema. Other treatment-related adverse events included fever, flu-like symptoms (including anorexia, nausea, and vomiting), and elevated liver enzyme tests. In general, these adverse events were reported by patients across all dose cohorts. The most common treatment-related adverse event, injection site reactions, was not unexpected and was manageable.

Elevated liver enzyme levels (AST, ALT, and GGT) occurred in one patient in the $700 \mu \mathrm{g}$ cohort, and three patients at the $930 \mu \mathrm{g}$ dose level. Two patients in the $930 \mu \mathrm{g}$ dose cohort experienced grade 3 elevated liver enzyme levels after the first two doses and this was determined to be the DLT and these patients discontinued treatment. In one patient, this adverse event was resolved within 13 days when liver functions returned to normal. The other patient died seven days after the second dose, from grade 4 multiple organ failure, which was considered to be due to disease progression rather than to VB4-845 administration. Of the four patients with elevated liver enzyme levels, three had the highest VB4-845 plasma concentration with maximum concentration $\left(\mathrm{C}_{\max }\right)$ values ranging from $18,809-49,363 \mathrm{pg} / \mathrm{mL}$. It is well documented that EpCAM is not expressed on adult hepatocytes but is detectable on bile duct epithelium (De Boer et al 1999; Schmelzer and Reid 2008). Patients with elevated liver enzyme levels did not present with any overt signs of liver damage or toxicity such as abdominal pain, tenderness, enlarged liver, jaundice, or even signs of decreased synthetic capacity of the liver due to severe liver damage such as coagulopathy or edema. Moreover, no indication of bile duct obstruction was observed, the predicted outcome with bile duct epithelial injury. As the patients with elevated enzymes, for whom follow-up was available, were asymptomatic and the levels were transient and reversible, the most likely explanation for the enzyme levels is an increase in physiologic activity of hepatocytes in response to drug clearance. Interestingly, the two patients treated at the highest dose level that did not exhibit any DLTs associated with an increase in liver enzymes had been pre-sensitized to ETA. This would suggest that the pre-existing antibody response may have afforded a degree of protection against hepatic changes attributed to circulating drug. 
Table 2 Patients reporting drug-related adverse events

\begin{tabular}{|c|c|c|c|c|c|c|}
\hline \multirow[t]{3}{*}{ Adverse event ${ }^{a}$} & \multicolumn{6}{|c|}{ Drug dose ( $\mu$ g/week) } \\
\hline & 100 & 200 & 330 & 500 & 700 & 930 \\
\hline & $\mathbf{N}=3^{b}$ & $\mathbf{N}=3^{\mathrm{b}, \mathrm{c}}$ & $\mathbf{N}=3^{b}$ & $\mathbf{N}=3^{\mathrm{d}}$ & $\mathbf{N}=\mathbf{3}^{\mathrm{b}}$ & $\mathbf{N}=\mathbf{5}^{\mathrm{b}, \mathrm{e}}$ \\
\hline Injection site redness ${ }^{f}$ & $\mathrm{I}(\mathrm{I})$ & I & & & $3(2)$ & 3 \\
\hline Injection site edema ${ }^{f}$ & $I(I)$ & & & I & $3(1)$ & 1 \\
\hline Injection site pain ${ }^{f}$ & 2 & & I & $\mathrm{I}(\mathrm{I})$ & 1 & I \\
\hline Injection site ulcer $^{f}$ & & & & I & & \\
\hline Fever & 1 & I & I & 1 & & 1 \\
\hline Elevated liver enzymes:AST,ALT & & & & & $\mathrm{I}(\mathrm{I})$ & $3(2)^{g}$ \\
\hline GGT & & & & & $I(I)$ & $I(I)$ \\
\hline Albumin & & & & & & I \\
\hline Alkaline phosphatase & & & & & $\mathrm{I}(\mathrm{I})$ & \\
\hline Creatinine & & & & & & I \\
\hline Anorexia & & & & & 3 & $I(I)$ \\
\hline Anemia & 1 & & & & & I \\
\hline Cancer pain & & I & & $I(I)$ & & \\
\hline Performance decrease & & & & & & $\mathrm{I}(\mathrm{I})$ \\
\hline Nausea & 1 & & & & 1 & \\
\hline Vomiting & & & & & 2 & \\
\hline Oral abscess & & & & I & & \\
\hline Headache & & & & I & & \\
\hline Pains in extremity & & & & I & & \\
\hline Tongue edema & & & & 1 & & \\
\hline Hematuria & 1 & & & & & \\
\hline Rigors (chills) & & & & 1 & & \\
\hline Asthenia (weakness) & & & & & & I \\
\hline Fatigue & & & & & & I \\
\hline
\end{tabular}

Notes: Numbers in parentheses represent the number of patients with grade 3 or 4 adverse events. ${ }^{2}$ Drug-related events which are possibly, probably or definitely related to the study drug. If a patient experienced an adverse event more than once, the event with the highest grade was tabulated. ${ }^{\circ}$ One patient discontinued due to death, deemed to not be treatment related. 'Treatment was discontinued at one patient's request. ${ }^{\mathrm{d}}$ Treatment was discontinued in one patient due to adverse event. ${ }^{\mathrm{e}}$ Treatment was discontinued in two patients due to adverse events. ${ }^{f}$ Local injection toxicity scale based on the injection site reaction event in $\mathrm{NCl}$ CTC version 2.0: pain, erythema and swelling of the skin or mucosa limited to the injection site and an area $\leq 3 \mathrm{~cm}$ adjacent to the tumor borders was classified as grade I; a similar reaction in an area $>3 \mathrm{~cm}$ and $\leq 6 \mathrm{~cm}$ adjacent to the tumor borders classified as grade 2. Extension of the area to $>6 \mathrm{~cm}$, or the presence of necrosis or ulceration that was severe or prolonged and required surgery, or arterial bleeding projecting from the wound which was self-limited with pressure defined grade 3 toxicity. Grade 4 injection toxicity was a life-threatening or chronically disabling local reaction or a hemorrhage. ${ }^{8 D L T}$.

Abbreviations: AST, aspartate aminotransferase; ALT, alanine aminotransferase; DLT, dose-limiting toxicity; GGT, gamma-glutamyltranspeptidase; NCI CTC, National Cancer Institute common toxicity criteria.

Although tumor bleeding was reported in some patients, none of these events were related to VB4-845. Two of the events occurred in nontarget tumors and the other two were in locations spatially separated from the injection site. Tumor bleeding in patients with advanced SCCHN is not unexpected (Sittel et al 2001). Four patients died during the course of the study, and one patient died after withdrawal from the study (withdrawal was due to DLT); however, none of the deaths were deemed treatment-related.

\section{Pharmacokinetics}

The pharmacokinetic parameters of VB4-845 in the patients' blood were analyzed to evaluate the release of the drug into circulation following IT injection. Patients with both lower and higher dose levels had detectable VB4-845 in the blood (Table 3). The mean $\mathrm{C}_{\text {max }}$ ranged from 10 to $14,997 \mathrm{pg} / \mathrm{mL}$, and the mean area under the curve $\left(\mathrm{AUC}_{0 \text {-last }}\right)$ ranged from
64 to $38,802 \mathrm{pg} / \mathrm{mL} \cdot \mathrm{h}$. Although there was a trend towards increased VB4-845 levels at higher dose levels with the exception of the $500 \mu \mathrm{g}$ dose, a high degree of variability was evident across each dose. In several patients VB4-845 was below the limits of detection $(<7 \mathrm{pg} / \mathrm{mL})$ at one or more time points; the high variability and nonlinear kinetics may be due to several factors such as vascularization, tumor size and antibody neutralization (see below). The mean $\mathrm{t}_{1 / 2}$ across all doses was $4.68 \pm 2.54$ hours suggesting a rapid clearance from the bloodstream. There was no evidence of accumulation of VB4- 845 between doses in any of the patients (data not shown).

\section{Immunogenicity}

The immunogenicity of VB4-845 was examined by analyzing the titers of anti-scFv and anti-ETA ${ }_{252-608}$ antibodies in blood samples of patients taken at various time points during the trial 
Table 3 Pharmacokinetics of VB4-845 dosing

\begin{tabular}{lllll}
\hline Dose level $(\mu \mathrm{g})$ & \# of patients & $\mathbf{C}_{\max }(\mathbf{p g} / \mathbf{m L})$ & AUC $_{0-\text { last }}(\mathbf{p g} / \mathbf{m L} \cdot \mathbf{h})$ & $\mathbf{t}_{1 / 2}{ }^{\mathbf{a}}(\mathbf{h})$ \\
\hline 100 & 2 & $1145 \pm 1619$ & $1955 \pm 2764$ & $3.38(\mathrm{I})$ \\
200 & 3 & $2090 \pm 1815$ & $6915 \pm 6072$ & $3.85 \pm 0.49(2)$ \\
330 & 3 & $1269 \pm 2199$ & $1886 \pm 3266$ & $1.84(\mathrm{I})$ \\
500 & 3 & $10 \pm 17$ & $64 \pm 111$ & $\mathrm{ND}^{\mathrm{b}}$ \\
700 & 3 & $10,936 \pm 9772$ & $4.45 \pm 1.00(2)$ \\
930 & 5 & $14,997 \pm 21,903$ & $38,802 \pm 38,955$ & $5.95 \pm 3.36(4)$ \\
\hline
\end{tabular}

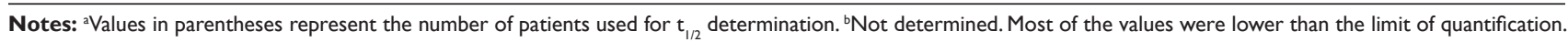
The inability to detect VB4-845 is most likely due to the presence of pre-existing anti-ETA antibodies found in 2 out of 3 patients in the $500 \mu g$ dose level. The lower and upper limits of quantification were $7 \mathrm{pg} / \mathrm{ml}(0.1 \mathrm{pM})$ and II $2 \mathrm{pg} / \mathrm{mL}(\mathrm{I} .6 \mathrm{pM})$, respectively with intra- and inter-day variability of $<25 \%$. Plasma pharmacokinetic variables were calculated based on all time points using WinNonlin Pro (version 4.1, Pharsight Corporation, Mountain View, CA, USA).

Abbreviations: AUC, area under the curve; ETA, Pseudomonas exotoxin A; ND, not determined.

(Table 4). Prior to treatment, 15 of 19 patients had antibodies to the exotoxin portion of the recombinant fusion protein. Over the course of VB4-845 treatment, an increasing number of patients developed anti-ETA ${ }_{252-608}$ responses, and by the end, of the study, all patients analyzed had detectable anti-ETA ${ }_{252-608}$ titers. Antibody titers beyond the limit of detection to the scFv portion of VB4-845 (anti-scFv) were not evident until day 15 but developed over the course of the study with 15 of 17 analyzable patients having detectable anti-scFv titers by the end of treatment (Day 28); the mean anti-scFv titers were considerably lower than the mean anti-ETA ${ }_{252-608}$ titers $(19,500$ vs. 124,100). For some patients the anti-ETA and anti-scFv titers remained elevated up to a month after dosing (Day 56).

Detectable antibody reactivity was observed against both the ETA and $\mathrm{scFv}$ moieties prior to initiating treatment (Day 1). Only the antibody reactivity against the ETA portion was considered noteworthy as the antibody titer was shown to be higher on Day 8 relative to Day 1 whereas the scFv titer was unchanged. Of the 15 patients that were presensitized to the ETA exotoxin, the plasma from seven elicited an inhibitory effect in a VB4-845-mediated cytotoxicity assay indicating the presence of neutralizing antibodies (data not shown). For six of the seven patients, the effect of neutralizing antibodies was concurrent with below detectable levels of
VB4-845 in patient plasma in the pharmacokinetic analysis. Even though all analyzable patients had eventually developed an immune response to VB4-845, the effect of neutralizing antibodies was only demonstrated in seven patients.

Although evaluation of efficacy was not a primary objective in this study, we obtained preliminary data that indicated a notable reduction (ten patients) or stabilization (four patients) of tumor growth in 16 EpCAM-positive patients based on radiographic and caliper measurements. Of the EpCAM positive patients that demonstrated a tumor response, four patients had complete clinical resolution of their tumors. An example of a complete response in one patient is seen in computed tomography scans and digital photographs (Fig. 1a-d). In addition, three out of the four patients with complete responses had regression or complete resolution of noninjected lesions adjacent to the injected tumors. One patient with dermal metastasis, which is rare and often associated with a poor prognosis in SCCHN, experienced resolution of noninjected cutaneous metastases (Fig. 1e-f). This patient presented with a lesion in the right submandibular region that was the principal injected tumor, along with cutaneous lesions; none of these cutaneous lesions were injected. The principal tumor achieved a complete response, and the cutaneous lesions visibly resolved.

Table 4 Antibody responses to the scFv and exotoxin portions of VB4-845

\begin{tabular}{|c|c|c|c|c|}
\hline \multirow[t]{2}{*}{ Day } & \multicolumn{2}{|c|}{ ETA response } & \multicolumn{2}{|c|}{ scFv response } \\
\hline & Patients $^{a}$ & Mean Titer $\left(\times 10^{-3}\right)$ & Patients $^{\mathbf{a}}$ & Mean Titer $\left(\times \mathrm{IO}^{-3}\right)$ \\
\hline $\mathrm{I}$ & $15 / 19$ & $2.5(1.2)$ & $6 / 19$ & $I(I)$ \\
\hline 8 & $7 / 19$ & $4.2(1.6)$ & $0 / 19$ & ND \\
\hline 15 & $13 / 18$ & $77.6(1.7)$ & $11 / 18$ & $5.7(\mathrm{I} .3)$ \\
\hline 22 & $16 / 18$ & $125.0(1.5)$ & $15 / 18$ & $12.4(1.4)$ \\
\hline 28 & $17 / 17$ & I24.I (I.5) & $15 / 17$ & $19.5(1.3)$ \\
\hline 56 & II/II & $181.3(1.5)$ & $9 / 11$ & I2.4 (I.4) \\
\hline
\end{tabular}

Notes: ${ }^{a}$ Number of patients with detectable response/number of patients evaluated. Mean titer is the calculated geometric mean of those patients with measurable titers on that given day. Values in parentheses are the corresponding standard error. Mean titer on day 8 was not determined as all values were below the limit of detection after subtracting Day I values.

Abbreviations: ETA, Pseudomonas exotoxin A; ND, not determined. 

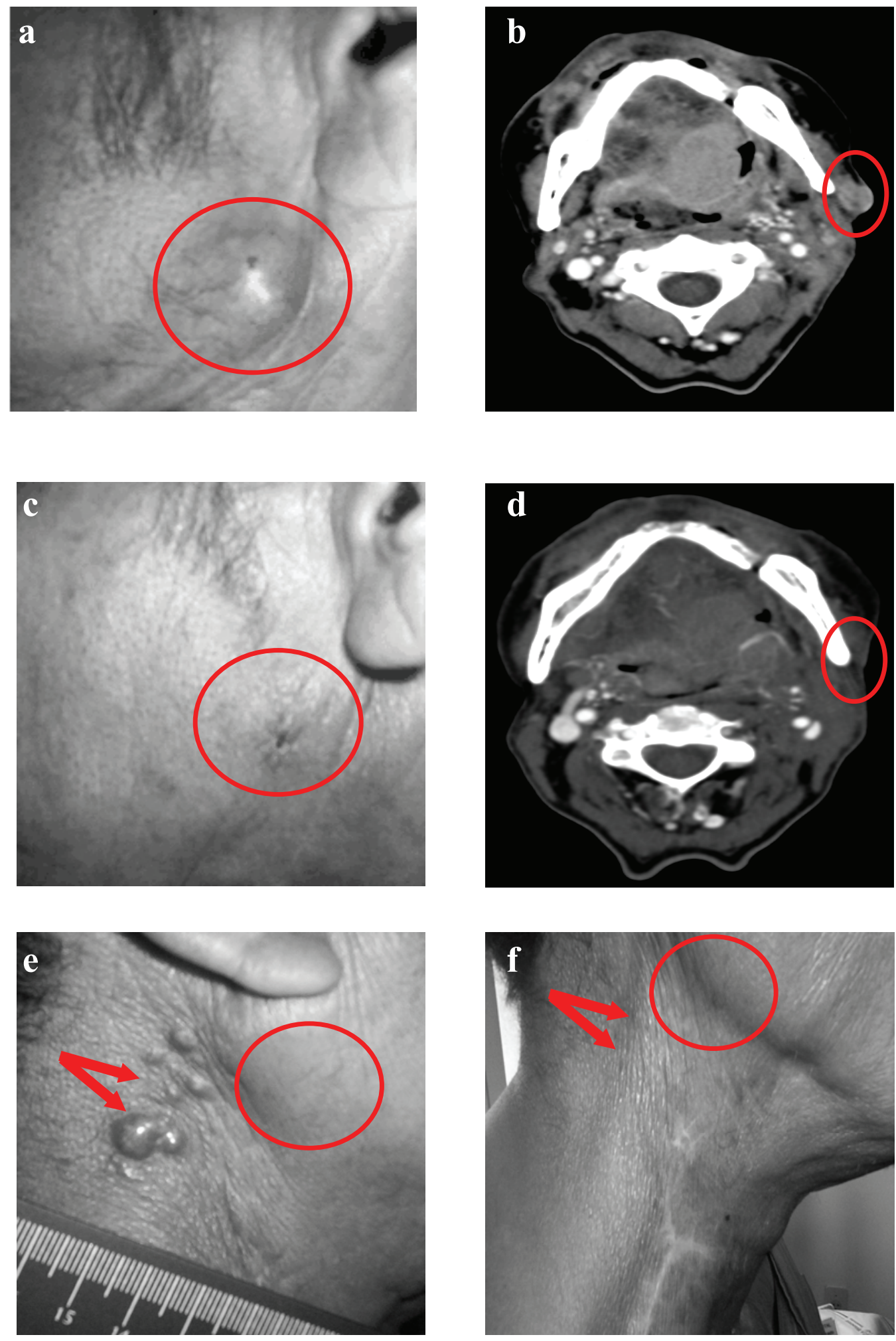

Figure I Resolution of targeted and nontargeted tumors in response toVB4-845 treatment. Digital photograph (a) and corresponding computed tomography scan (b) of the targeted tumor lesion in patient A at baseline. Circled regions denote location of the targeted tumor. Digital photograph (c) and corresponding computed tomography scan (d) of the VB4-845 treated tumor lesion in patient A at final visit. Circled regions denote location of the targeted tumor as originally seen at baseline. Digital photograph of the location of dermal metastases (arrows) and targeted tumor (circled region) in patient $B$ at baseline (e) and at final visit (f), respectively. 


\section{Discussion}

This phase I trial of the safety and tolerability of VB4-845 IT injections administered once weekly for four consecutive weeks indicated that VB4-845 was safe and well-tolerated and that adverse events were manageable. The MTD was established at $930 \mu \mathrm{g}$ given once per week as indicated by a DLT of elevated liver enzyme levels in two patients at this dose level. The most common treatment-related adverse events were injection site reactions, fever, gastrointestinal disorders, and transiently elevated liver enzymes. Similar results were observed in another clinical trial with VB4-845 administered through IT injections on a daily dosing schedule (five consecutive days) with two cycles of treatment, followed by 23 days of rest (manuscript in preparation). The weekly mode of administration in the current study was preferred over a daily dosing regimen, as it is more convenient for patients.

IT administration was chosen to reduce the possibility of vascular leak syndrome, a common side effect with some immunotoxins (Siegall et al 1997), and to lessen the effect of circulating antibodies. In this study, approximately half of the patients tested had detectable levels of VB4-845 in their blood following IT administration with all patients developing antibodies to VB4-845 over the course of the trial. This suggests that in some patients, the drug clears from the tumor into the systemic circulation. Pseudomonas exotoxin is immunogenic, and therefore an antigenic response to VB4-845 was anticipated. By the end of the study all patients had anti-ETA ${ }_{252-608}$, and most patients had anti-scFv responses. The anti-scFv titers were generally lower than the anti-ETA titers and may have resulted from a carrier effect because of its proximity to the exotoxin. The presence of anti-ETA antibodies in a high proportion of patients prior to treatment is possibly due to previous exposure of the patient to Pseudomonas (Fitzgerald et al 1989), which may result in neutralization and a reduction of drug in circulation. Although the antibody response was neutralizing in some patients, low or nondetectable levels of VB4-845 could not be attributed to neutralizing antibodies in other patients. Other potential reasons for the lack of detection of VB4-845 in the bloodstream of some patients are that only low levels of drug move from the tumor into the systemic circulation, or that the rapid clearance of the drug from the systemic circulation of some patients reduces levels to below the limits of detection. High variability from patient to patient in pharmacokinetic parameters of intratumorally administered biologic immune activators has been previously noted (van Herpen et al 2003, 2004).
Preliminary efficacy data indicated a notable reduction or stabilization of tumor growth in EpCAM-positive patients based on radiographic and caliper measurements. This antitumor activity was seen despite the fact that dosing in this study was not optimized for therapeutic benefit. These results complement data obtained in an earlier trial with a daily dosing schedule, where VB4-845 also showed antitumor activity (manuscript in preparation). In some instances, antitumor effects were observed in adjacent and distal noninjected tumors. In particular, one patient with dermal metastasis, a condition often associated with late stage disease and poor prognosis (Cole et al 1995; Pitman et al 1999; Yoskovitch et al 2001; Dequanter et al 2005), exhibited complete resolution of these secondary tumors.

Currently therapeutic options for the treatment of SCCHN are limited, and survival rates after failure of first line treatment options are very low (León et al 2005). A novel alternative currently under investigation for the treatment of SCCHN is the application of photodynamic therapy (PDT). PDT provides a noninvasive treatment approach to tumor cell killing through the activation of photosensitizing drugs and the release of singlet molecular oxygen (Jerjes et al 2007). Clinical results achieved with PDT in the treatment of SCCHN to-date have been comparable with or better than standard treatment protocols but without the associated morbidity. However, as the photosensitizer is generally administered systemically, the drug is free to accumulate throughout the body and as such can lack the specific targeting of other methods.

Monoclonal antibody based therapies provide a targeted approach that can be applied in combination with traditional therapies. For example, a phase III study combining Cetuximab and radiotherapy significantly prolonged progressionfree survival in patients with locally advanced inoperable SCCHN (Bonner et al 2006), and Cetuximab has also shown antitumor activity in two phase II trials in combination with chemotherapy (Baselga et al 2005; Herbst et al 2005). However, combination therapy has yet to establish efficacy above the current standard of chemoradiotherapy (Posner and Wirth 2006).

This study has established an MTD for weekly dosing of VB4-845, as well as demonstrating that repeated IT delivery of VB4-845 was not limited by immunogenicity and was well tolerated. Preliminary efficacy results also indicated a positive outcome to VB4-845 therapy in EpCAM-positive patients. These promising results will be extended in a larger placebo-controlled clinical trial with optimized dosing to demonstrate the effectiveness of VB4-845 as a therapeutic agent for the treatment of SCCHN. 


\section{Disclosure}

Michèle Rasamoelisolo, Joycelyn Entwistle, Jeannick Cizeau, Denis Bosc, Wendy Cuthbert, Mark Kowalski, Maureen Spearman, Glen MacDonald, and Nick Glover are officers or employees of Viventia Biotech Inc. which has direct financial interest in the subject matter discussed in this manuscript.

\section{Acknowledgments}

Previously presented at the 41st Annual Meeting of the American Society of Clinical Oncology, May 13-17, 2005, Orlando, Florida. We would like to thank Dr. Barry Wenig, Evanston Northwestern Healthcare, Dimitri Fitsialos, Joseé Quenneville, Jacinthe Guindon, Caroline Woods, Jennifer Brown, and Heather Graham for their contributions to the review and preparation of the manuscript, and Dr. Philippa Bridge-Cook for drafting assistance. We gratefully acknowledge the contribution of the Dr. Rodrigo C Guimarães, Dr. Miriam HH Federico, Dr. Ulisses R Nicolau, and Dr. Carlos HE Barrios for their participation in the study.

\section{References}

Azemar M, Djahansouzi S, Jäger E, et al. 2003. Regression of cutaneous tumor lesions in patients intratumorally injected with a recombinant single-chain antibody-toxin targeted to Erb2/HER2. Breast Can Res Treat, 82:155-64.

Balzar M, Winter MJ, de Boer CJ, et al. 1999. The biology of the 17-1A antigen (Ep-CAM). J Mol Med, 77:699-712.

Baselga J, Trigo JM, Bourhis J, et al. 2005. Phase II multicenter study of the antiepidermal growth factor receptor monoclonal antibody cetuximab in combination with platinum-based chemotherapy in patients with platinum-refractory metastatic and/or recurrent squamous cell carcinoma of the head and neck. J Clin Oncol, 23:5568-77.

Blick SK, Scott LJ. 2007. Cetuximab, a review of its use in squamous cell carcinoma of the head and neck and metastatic colorectal cancer. Drugs, 67:2585-607.

Bonner JA, Harari PM, Giralt J, et al. 2006. Radiotherapy plus cetuximab for squamous-cell carinoma of the head and neck. $N$ Engl J Med, 354:567-78.

Bossi P, Locati L, Licitra L. 2007. Biological agents in head and neck cancer. Expert Rev Anticancer Ther, 7:1643-50.

Brown J, Cizeau J, Bosc D, et al. 2005. A preclinical profile of VB4-845 a recombinant immunotoxin for targeting head and neck cancer. 96th Annual Meeting of the American Association of Cancer Research.

Cole RD, McGuirt F. 1995. Prognostic significance of skin involvement from mucosal tumors of the head and neck. Arch Otolaryngol Head Neck Surg, 121:1246-8.

De Boer CJ, Van Krieken JHJM, Janssen-van Rhijn CM, et al. 1999. Expression of Ep-CAM in normal, regenerating, metaplastic, and neoplastic liver. J Pathology, 188:201-6.

Dequanter D, Mboti FB, Lothaire P, et al. 2005. Skin metastases from a head and neck carcinoma: a prognostic factor? B-Ent, 1:113-15.

Di Paolo C, Willuda J, Kubetzko S, et al. 2003. A recombinant immunotoxin derived from a humanized epithelial cell adhesion molecule-specific single-chain antibody fragment has potent and selective antitumor activity. Clin Cancer Res, 9:2837-48.

Fitzgerald D, Pastan I. 1989. Targeted toxin therapy for the treatment of cancer. J Natl Cancer Inst, 81:1455-63.
Gebbia V, Giuliani G, Valori VM, et al. 2007. Cetuximab in squamous cell head and neck carcinomas. Ann Oncol, 18(supp6):vi5-vi7.

Haddad R, Allen A, Wirth L, et al. 2006. Integrating novel agents into the curative treatment of head and neck cancer. Expert Rev Anticancer Ther, 6:157-9.

Herbst RS, Arquette M, Shin DM, et al. 2005. Phase II multicenter study of the epidermal growth factor receptor antibody cetuximab and cisplatin for recurrent and refractory squamous cell carcinoma of the head and neck. J Clin Oncol, 23:5578-87.

Jerjes W, Upile T, Betz CS, et al. 2007. The application of photodynamic therapy in the head and neck. Dent Update, 34:478-86.

Laimer K, Fong D, Gastl G, et al. 2008. EpCAM expression in squamous cell carcinoma of the oral cavity: frequency and relationship to clinicopathologic features. Oral Oncol, 44:72-7.

Le Tourneau C, Faivre S, Siu LL. 2007. Molecular targeted therapy of head and neck cancer: review and clinical development challenges. Eur $J$ Cancer, 43:2457-66.

León X, Hitt R, Constenla M, et al. 2005. A retrospective analysis of the outcome of patients with recurrent and/or metastatic squamous cell carcinoma of the head and neck refractory to a platinum-based chemotherapy. Clin Oncol (R Coll Radiol), 17:418-24.

Litvinov SV, Velders MP, Bakker HA, et al. 1994. Ep-CAM: a human epithelial antigen is a homophilic cell-cell adhesion molecule. $J$ Cell Biol, 125:437-46.

Litvinov SV, Balzar M, Winter MJ, et al. 1997. Epithelial cell adhesion molecule (Ep-CAM) modulates cell-cell interactions mediated by classic cadherins. J Cell Biol, 139:1337-48.

MacDonald GC, Glover N. 2005. Effective tumor targeting: strategies for the delivery of armed antibodies. Curr Opin Drug Discov Devel, $8: 177-83$

Pastan I, Hassan R, Fitzgerald DJ, et al. 2006. Immunotoxin therapy of cancer. Nat Rev Cancer, 6:559-65.

Pastan I, Hassan R, Fitzgerald DJ, et al. 2007. Immunotoxin treatment of cancer. Ann Rev Med, 58:221-37.

Perentesis JP, Miller SP, Bodley JW. 1992. Protein toxin inhibitors of protein synthesis. Biofactors, 3:173-84.

Pitman KT, Johnson JT. 1999. Skin metastases from head and neck squamous cell carcinoma: incidence and impact. Head Neck, 21:560-65.

Posner M. 2007. Evolving strategies for combined-modality therapy for locally advanced head and neck cancer. Oncologist, 12:967-74.

Posner MR, Wirth LJ. 2006. Cetuximab and radiotherapy for head and neck cancer. N Engl J Med, 354:634-36.

Rand RW, Kreitman RJ, Patronas N, et al. 2000. Intratumoral administration of recombinant circularly permuted interleukin-4-Pseudomonas exotoxin in patients with high grade glioma. Clin Can Res, 6:2157-65.

Robert F, Ezekiel MP, Spencer SA, et al. 2001. Phase 1 study of antiepidermal growth factor receptor antibody cetuximab in combination with radiation therapy in patients with advanced head and neck cancer. J Clin Oncol, 19:3234-43.

Schmelzer F, Reid LM. 2008. EpCAM expression in normal, non-pathological tissues. Front Biosci, 13:3096-100.

Siegall CB, Liggitt D, Chace D, et al. 1997. Characterization of vascular leak syndrome induced by the toxin component of Pseudomonas exotoxin-based immunotoxins and its potential inhibition with nonsteroidal anti-inflammatory drugs. Clin Cancer Res, 3:339-45.

Sittel C, Gossmann A, Jungehulsing M, et al. 2001. Superselective embolization as palliative treatment of recurrent hemorrhage in advanced carcinoma of the head and neck. Ann Otol Rhinol Laryngol, 110:1126-28.

Stoecklein NH, Siegmund A, Scheunemann P, et al. 2006. EpCAM expression in squamous cell carinoma of the esophagus: a potential therapeutic target and prognostic marker. BMC Cancer, 6:165-73.

van Herpen CM, Huijbens R, Looman M, et al. 2003. Pharmacokinetics and immunological aspects of a phase Ib study with intratumoral administration of recombinant human interleukin-12 in patients with head and neck squamous cell carcinoma: a decrease of T-bet in peripheral blood mononuclear cells. Clin Can Res, 9:2950-56. 
van Herpen CM, Looman M, Zonneveld M, et al. 2004. Intratumoral administration of recombinant interleukin 12 in head and neck squamous cell carcinoma patients elicits a T-helper 1 profile in the locoregional lymph nodes. Clin Can Res, 10:2626-35.

von Mehren M, Adams GP, Weiner LM. 2003. Monoclonal antibody therapy in cancer. Ann Rev Med, 54:343-69.

Went PT, Lugli A, Meier S, et al. 2004. Frequent EpCam protein expression in human carcinomas. Hum Pathol, 35:122-28.

Winter MJ, Nagtegaal ID, van Krieken JH, et al. 2003. The epithelial cell adhesion molecule (Ep-CAM) as a morphoregulatory molecule is a tool in surgical pathology. Am J Pathol, 163:2139-48.
Yanamoto S, Kawasaki G, Yoshitomi I, et al. 2007. Clinicopathologic significance of EpCAM expression in squamous cell carcinoma of the tongue and its possibility as a potential target for tongue cancer gene therapy. Oral Oncol, 43:869-77.

Yoskovitch A, Hier MP, Okrainec A, et al. 2001. Skin metastases in squamous cell carcinoma of the head and neck. Otolaryngol Head Neck Surg, 124:248-52. 